\title{
Spreading-Hopping Tradeoff in Wideband Ad-hoc Communications
}

\author{
Sudhir Srinivasa and Syed Ali Jafar \\ Electrical Engineering and Computer Science \\ University of California Irvine, Irvine, CA 92697-2625 \\ Email: sudhirs@uci.edu,syed@ece.uci.edu
}

\begin{abstract}
We explore the tradeoff between hopping and spreading in a wideband spread spectrum transmitter-receiver link in an ad-hoc network with multi-user interference. The receiver is assumed to track the user's channel, spreading signature and the overall SINR. Using expressions for the ergodic capacity of the link, we determine the amount of spreading required that maximizes the link capacity. As a comparison we also discuss optimal spreads in centralized architectures where the receiver has interference knowledge. While we find that in general a combination of spreading and hopping may achieve higher throughputs than either pure spreading or pure hopping, we prove that pure hopping and pure spreading are optimal strategies for ad-hoc networks at low SINR and for centralized networks at all SINR, respectively. Numerical results suggest that in an ad-hoc network, spreading is beneficial at high SNR.
\end{abstract}

\section{INTRODUCTION}

The growing popularity of ad-hoc networks in wideband channels can be attributed to their ease and flexibility of deployment and the minimal infrastructure requirement. Contrary to cellular architectures where the base station has channel and interference knowledge, communication in ad-hoc and sensor networks is decentralized and is expected to occur without any collusion between the different transmitter-receiver pairs. Due to the dynamic nature of the ad-hoc network, the receiver usually does not have knowledge of the multi-user interference. In such scenarios, spread spectrum architectures like frequency hopping or direct sequence techniques are attractive and robust communication solutions since they allow for simultaneous transmissions without the need for any interaction between the different users in the system.

The literature for the information theoretic study of spreading in wideband cellular and ad-hoc systems is extensive [1]-[9]. In [3], [4] the spreading-coding tradeoff problem is considered and a general approach to optimize the tradeoff is provided. [5], [6] investigate the optimal spreading factor from a capacity perspective accommodating both channel estimation and training into the capacity formulation. Upper and lower bounds on the channel capacity are used to determine the bandwidth range for which spreading is beneficial. While the optimal capacity achieving codes are viewed as a generalized version of a DS-CDMA scheme in [5], [6], our definition of spreading is specialized to conventional DSCDMA and FH systems. We regard spreading as an operation that expands the number of dimensions (frequency slots) used by the signal without expanding the rank of the signal space (unit rank for conventional CDMA). Mathematically, the transmitted signal covariance matrix for a conventional multicarrier CDMA system is unit rank, while the formulation of [5], [6] allows full rank signals. Using the probability of error as a performance measure, [8] compares frequency hopping and direct sequence spreading in frequency selective channels with Gaussian frequency correlation. [2] analyzes the 'transmission capacity' of ad-hoc CDMA networks with successive interference cancellation, defined as the maximum density of simultaneous transmissions that can be supported at a given signal to interference ratio. We emphasize here that the interplay between spreading and hopping in wideband ad-hoc networks has not been investigated from a ergodic capacity perspective.

In a fading link with interference, it is well known that a constant channel provides a higher capacity than a timevarying one [10], [11]. This is due to the logarithmic dependence of the capacity on the signal to interference ratio (SINR). However, this dependence, as we see in Section II also results in higher throughputs with time-varying interference power than with constant interference power. Therefore while a constant channel gain power is beneficial, constant interference power hurts the link capacity. In a spreading system, the despreading at the receiver essentially makes the channel constant by exploiting the frequency diversity available. On the downside it also 'averages' out the interference. On the other hand, frequency hopping presents the user with timevarying interference which is favorable. However, since only one frequency slot is occupied during any transmission, there is no diversity, and the receiver encounters a changing channel. Therefore with either pure hopping or pure spreading, it is not possible to satisfy the conflicting requirements of a constant channel and fluctuating interference power. It is this tradeoff between spreading and hopping that we are concerned with in this work.

In this paper, we consider a spread spectrum link in a wideband ad-hoc network. The receiver is assumed to have knowledge of only the link's channel and the SINR and cannot perform multi-user detection. Providing capacity expressions for the link capacity, we identify and characterize the tradeoff between spreading and hopping in such systems. We exploit this tradeoff to optimize the spread that maximizes the user capacity. We begin with a discussion on the spreading-hopping tradeoff in Section II.

\section{TO SPREAD OR NOT TO SPREAD}

It is well documented that the ergodic capacity of a fading channel with perfect CSIR and no CSIT is lower than that 
of the corresponding AWGN channel with the same average channel power gain, i.e., time-varying fading diminishes system capacity [10], [11]. The same behavior is evidenced in a system with multi-user interference. To illustrate, we consider a single point to point link in an ergodic fading channel. Let the channel between the transmitter and receiver be denoted by $H$. The noise at the receiver is assumed to be zero mean Gaussian with power $N$. Let the interference seen by the receiver also be Gaussian with power $I$. We assume that the interference power $I$ is itself random and model it as an ergodic stochastic process. The interference will therefore be drawn from a Gaussian distribution with some time varying power. The receiver has channel $(H)$ and interference $(I)$ knowledge while the transmitter has no channel information. The ergodic capacity of such a link is given by

$$
C_{\text {link }}=\mathrm{E}_{H} \mathrm{E}_{I}\left[\log \left(1+\frac{P|H|^{2}}{I+N}\right)\right],
$$

where $P$ is the transmit power constraint. Since the $\log ()$ function is concave [12], we can write

$$
C_{\text {link }} \leq \mathrm{E}_{I}\left[\log \left(1+\frac{P \mathrm{E}_{H}\left[|H|^{2}\right]}{I+N}\right)\right]
$$

Note that the $\mathrm{E}_{H}\left[|H|^{2}\right]$ term in equation (2) can be thought of as arising due to diversity at the receiver. This diversity helps in mitigating the channel variations and consequently increases the link capacity. Equation (2) establishes that a channel with a constant gain $\left(\mathrm{E}_{H}\left[|H|^{2}\right]\right)$ yields a higher throughput than one whose gain is time-varying $\left(|H|^{2}\right)$.

Note, however, that we have only considered the effect of channel variations on the capacity so far and ignored the influence of the fluctuations in the interference power on the capacity. It is not clear whether, as in the case of the channel, a constant interference power yields a higher capacity than when the interference power is varying. Since $\log \left(1+\frac{1}{x}\right)$ is convex in $x$, applying Jensen's inequality [12] to equation (1) yields

$$
C_{\text {link }} \geq \mathrm{E}_{H}\left[\log \left(1+\frac{P|H|^{2}}{\mathrm{E}_{I}[I]+N}\right)\right] .
$$

The inequality in equation (3) brings out an interesting result - it shows that the link capacity is higher if the interference power is changing than when it stays constant with the same average power during all transmissions. Therefore from an ergodic link capacity perspective, variable interference power is preferred. Contrast this with the inequality from equation (2), where a constant channel power gain is favored. We summarize the observations so far thus: while averaging the channel power $\left(\mathrm{E}_{H}\left[|H|^{2}\right]\right)$ helps increase the link capacity, averaging the interference power $\left(\mathrm{E}_{I}[I]\right)$ decreases it.

Results from the above discussion provide useful insights in scenarios where there is an averaging of the interference or channel powers. An interesting example is a multi-slot wideband channel in an ad-hoc network. Consider a certain user spreading over $d(d \geq 1)$ slots with independent channel gains $H_{n}$ (for $1 \leq n \leq d$ ). We denote the interference power seen by the user in the $d$ slots by independent random variables $I_{m}$ (with $1 \leq m \leq d$ ) for simplicity. The receiver is assumed to have no information about the interference in the $d$ slots and is consequently unable to perform successive interference cancellation. We show in Section IV-B that the capacity of the user in such a system is given by

$$
C_{a d h o c}(d)=\mathrm{E}_{\mathbf{H}} \mathrm{E}_{\mathbf{I}} \log \left[1+\frac{\frac{P}{d} \sum_{n=1}^{d}\left|H_{n}\right|^{2}}{N+\frac{1}{\|\mathbf{H}\|^{2}} \sum_{m=1}^{d}\left|H_{m}\right|^{2} I_{m}}\right] .
$$

Notice from equation (4) that despreading the signal $(d>1)$ averages the channel power gains because of the frequency diversity obtained. But at the same time, it also averages the interference in the $d$ slots. If the user instead randomly hops between available frequency slots $(d=1)$, it can be seen from equation (4) that the receiver sees time-varying interference. However since only one slot is occupied every transmission, there is no diversity and the averaging in the numerator is absent. This is the source of the spreading-hopping tradeoff mentioned in Section I.

The issue of whether or not to spread therefore becomes especially relevant in an ad-hoc spread spectrum scenario since $b$ oth the interference and the channel power gains are averaged. In this work we are specifically interested in how much a user in an ad-hoc network should spread its signal (i.e., value of $d$ ) so that it achieves the highest throughput possible. In other words, we seek answers and insights into the following questions:

- Does spreading increase the ergodic capacity of the communicating user?

- If so, what is the optimal value $d^{*}\left(d^{*} \geq 1\right)$ of the user spread $d$ ?

From the previous discussion, it is intuitive to expect that in such a case a hybrid spreading-hopping scheme could offer higher data rates than either complete spreading or complete hopping. We make this intuition more precise by providing information theoretic insights into if and when spreading and/or hopping is appropriate in an ad-hoc network. A formal system model description in Section III begins our discussion.

\section{SySTEM MODEL}

We consider a single transmitter-receiver pair transmitting signals simultaneously with other users over a wide band channel in an ad-hoc network. The available channel bandwidth is divided into $T(T>>1)$ coherence bandwidth slots so that the fading channel appears as flat fading on each slot. During each channel use, the transmitter chooses $d$ independent frequency slots and spreads its signal over all the $d$ slots $(1 \leq d \leq T)$ using a spreading code.

Let the transmitter input be denoted by $X$, with $X$ spread over $d(d \geq 1)$ distinct and independent frequency slots as shown in Figure 1. The spreading code at the transmitter is given by $\mathbf{C}=\frac{1}{\sqrt{d}} e^{j \boldsymbol{\theta}}$, where $\boldsymbol{\theta}$ is a $(d \times 1)$ vector with elements $\theta_{i} \sim \mathcal{U}(0,2 \pi)$. The channel in the $d$ slots is specified by the vector $\mathbf{H}=\left[\begin{array}{llll}H_{1} & H_{2} & \cdots & H_{d}\end{array}\right]$, where the $H_{i}$ are independent and identically distributed Rayleigh variables. The power constraint at the transmitter is $\mathrm{E}\left[|X|^{2}\right] \leq P$. 


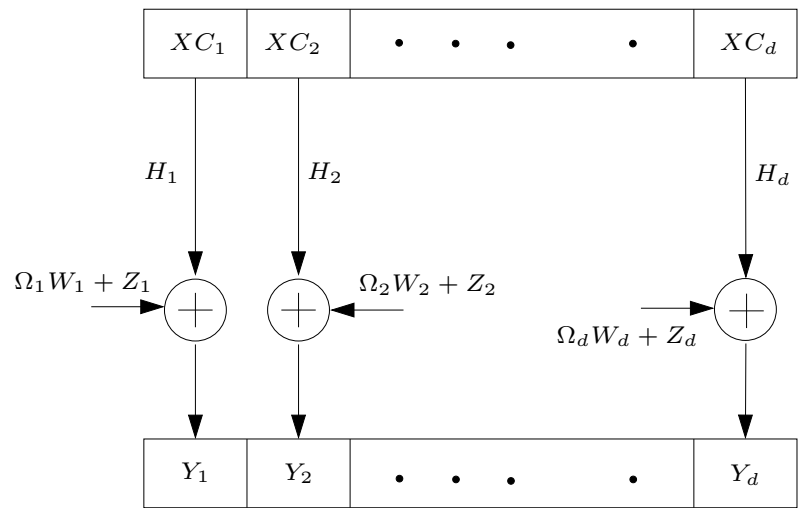

Fig. 1: System Model

In each slot, the user's signal not only encounters white noise at the receiver but also the interference caused by other users in the system occupying the same slot. We assume an unresponsive Gaussian interference model where the interference power in the $i^{t h}$ slot is given by $\Omega_{i}^{2}=P \alpha \lambda_{i}$, where $\lambda_{i}$ is a random variable reflecting the number of interfering users in the same slot. $\alpha P$ is a measure of the power of the interferers in the system relative to the communicating user $(0 \leq \alpha \leq 1)$, it can be thought of as a parameter signifying the distance of the interferers in the system from the communicating user. We will not constrain the $\lambda_{i}$ to follow any specific distribution in our analysis. The Gaussian interference in the $d$ slots will be represented by $\Omega \mathbf{W}$ where $\mathbf{W} \sim \mathcal{C N}\left(\mathbf{0}, \mathbf{I}_{d}\right)$ is a $(d \times 1)$ circularly symmetric Gaussian vector and $\Omega=\operatorname{diag}\left[\begin{array}{llll}\Omega_{1} & \Omega_{2} & \cdots & \Omega_{d}\end{array}\right]$. The unit variance white Gaussian noise at the receiver will be represented by the $(d \times 1)$ complex vector $\mathbf{Z} \sim \mathcal{C N}\left(\mathbf{0}, \mathbf{I}_{d}\right)$.

Let $\mathbf{Y}$ denote the $(d \times 1)$ received vector. The input-output relationship for the user can be written as

$$
\begin{aligned}
\mathbf{Y} & =(\mathbf{H} \odot \mathbf{C}) X+\mathbf{\Omega} \mathbf{W}+\mathbf{Z} \\
& =\frac{1}{\sqrt{d}}\left(\mathbf{H} \odot e^{j \boldsymbol{\theta}}\right) X+\mathbf{\Omega} \mathbf{W}+\mathbf{Z},
\end{aligned}
$$

where the $\odot$ operator denotes Hadamard multiplication. Note that since $\mathbf{H}$ consists of i.i.d Rayleigh elements, $\left(\mathbf{H} \odot e^{j \boldsymbol{\theta}}\right)$ has the same distribution as $\mathbf{H}$. We therefore write

$$
\mathbf{Y} \cong \frac{1}{\sqrt{d}} \mathbf{H} X+\boldsymbol{\Lambda} \mathbf{N}
$$

where $\cong$ signifies statistical equivalence. In equation (5), since the interference $\Omega \mathbf{W}$ and noise $\mathbf{Z}$ are independent, their sum is represented by a single term $\Lambda \mathrm{N}$ in equation (6), where $\mathbf{N} \sim \mathcal{C N}\left(\mathbf{0}, \mathbf{I}_{d}\right)$ is a complex Gaussian random variable and $\boldsymbol{\Lambda}=\operatorname{diag}\left[\Lambda_{1} \Lambda_{2} \cdots \Lambda_{d}\right]$ is a diagonal matrix representing the interference plus noise powers in the $d$ slots. To keep the same interference and noise power in each slot as in equation (5), we have to set $\Lambda_{i}=\sqrt{1+\Omega_{i}^{2}}=\sqrt{1+P \alpha \lambda_{i}}$. Equation (6) will be used as the channel model definition in subsequent sections.
We assume that the transmitter has no channel knowledge, i.e., there is no feedback from the receiver to the transmitter. The absence of channel information at the transmitter precludes any kind of power adaptation across the $d$ slots the user therefore spreads its data uniformly over all the $d$ frequency slots with a power $\frac{P}{d}$ in each slot.

While the main focus of this paper is on ad-hoc systems, we also consider centralized (cellular) systems in order to compare the optimal user spreads in the two scenarios. Note that the input-output relationship of equation (6) is also applicable for a centralized system. The differences between the two lie in the amount of receiver knowledge and the strategy used at the receiver to despread the signals in the $d$ slots. We now deliberate on these differences between cellular and ad-hoc systems.

1) Centralized scenarios: In a centralized uplink, we assume that the receiver tracks not only the channel of the communicating user and the spreading sequence of the user but also the interference power distributions in all the $d$ slots the user spreads its signal. In other words the receiver has access to both $\mathbf{H}$ and $\boldsymbol{\Lambda}$. This knowledge at the receiver allows it to optimally combine the signals in the $d$ slots as in maximum ratio combining (MRC).

2) Adhoc Scenarios: In an ad-hoc receiver, since there is no collaboration between the users, the receiver can only track the user's channel and the effective instantaneous post-spreading interference plus noise power $\frac{\left\|\mathbf{H}^{\dagger} \boldsymbol{\Lambda}\right\|^{2}}{\|\mathbf{H}\|^{2}}$. Note that the receiver is not aware of the interference powers in each of the individual slots $\Lambda_{n}$. The signals in the $d$ slots are therefore combined with $\frac{\mathbf{H}}{\|\mathbf{H}\|}$ as the combining weight vector.

Using the definitions and expressions obtained in this section, we now provide capacity expressions in both cellular and ad-hoc scenarios.

\section{CAPACITY EXPRESSIONS}

In this section, we consider the capacity optimization problem for the ad-hoc link of Section III. As a comparison we also provide capacity expressions for the corresponding centralized (cellular) link described in Section III-1. In both the centralized and ad-hoc cases we analyze the optimal user spreads that maximize the ergodic capacity. We begin with the centralized case.

\section{A. Centralized Links}

Consider a typical centralized uplink defined by the inputoutput relationship of equation (6). The interference-aware receiver at the base station combines the received signals in the different slots proportional to the signal to interference and noise ratio in each slot. The mutual information between the input $X$ and output $\mathbf{Y}$ is given by

$$
\begin{aligned}
\mathbb{I}(X ; \mathbf{Y} \mid \mathbf{H}) & =\mathrm{E}_{\mathbf{H}}[\mathbb{H}(\mathbf{Y})-\mathbb{H}(\mathbf{Y} \mid X, \mathbf{H})] \\
& =\mathrm{E}_{\mathbf{H}}[\mathbb{H}(\mathbf{H} X+\Lambda \mathbf{N})-\mathbb{H}(\Lambda \mathbf{N})]
\end{aligned}
$$

It can be shown that the mutual information in this case is maximized when the input $X$ is Gaussian and that the capacity is given by 


$$
C_{\text {cellular }}(d)=\mathrm{E}_{\mathbf{H}} \mathrm{E}_{\boldsymbol{\Lambda}} \log _{2}\left[1+\frac{P}{d} \sum_{i=1}^{d} \frac{\left|H_{i}\right|^{2}}{\Lambda_{i}^{2}}\right] .
$$

Note that in a centralized link, equation (6) represents a single input multiple output (SIMO) system with channel and interference knowledge at the receiver. The capacity expression in equation (7) therefore corresponds to maximum ratio combining (despreading) at the receiver.

The $H_{i}$ and $\Lambda_{i}$ are assumed to be independent. Using Jensen's inequality on equation (7) yields

$$
C_{\text {cellular }}(d) \leq \log _{2}\left[1+P \mathrm{E}_{H_{1}} \mathrm{E}_{\Lambda_{1}}\left[\frac{\left|H_{1}\right|^{2}}{\Lambda_{1}^{2}}\right]\right] .
$$

The RHS of equation (8) is achieved when the user spreads over a large number of slots i.e. $d \rightarrow \infty$ (by the Law of large numbers). Therefore in a centralized scenario, capacity is maximized when the user spreads over all the available slots.

The same is true in a multi-slot OFDM scenario where the user transmits $d$ independent streams with equal powers $\frac{P}{d}$ along $d$ parallel slots. Since each slot carries independent data, the capacity of the link is just the sum of the capacities in the individual links. We therefore have

$$
C_{O F D M}(d)=d \mathrm{E}_{H_{1}} \mathrm{E}_{\Lambda_{1}} \log _{2}\left[\left[1+\frac{P}{d} \frac{\left|H_{1}\right|^{2}}{\Lambda_{1}^{2}}\right]\right] .
$$

Note that the RHS of equation (9) is in the form $x \log \left(1+\frac{a}{x}\right)$ with $a \geq 0$, which does not decrease with $x$. Consequently, the optimal spread in this case would be to use as many slots as possible, i.e., spread over all the available slots.

Therefore for centralized links, regardless of the SINR, pure spreading over all the available slots offers higher throughputs compared to pure hopping or any hybrid spreading-hopping scheme.

\section{B. Ad-hoc Links}

In ad-hoc links, the receiver has no knowledge of the interference in any of the slots. Therefore the receiver will use $\mathbf{H}^{\dagger}$ as the combining vector, so that

$Y^{\prime}=\frac{\mathbf{H}^{\dagger}}{\|\mathbf{H}\|} \mathbf{Y}=\frac{1}{\sqrt{d}} \frac{\mathbf{H}^{\dagger}}{\|\mathbf{H}\|} \mathbf{H} X+\frac{\mathbf{H}^{\dagger}}{\|\mathbf{H}\|} \boldsymbol{\Lambda} \mathbf{N}=\frac{\|\mathbf{H}\|}{\sqrt{d}} X+\boldsymbol{\phi}^{\dagger} \boldsymbol{\Lambda} \mathbf{N}$,

where $\phi=\frac{\mathbf{H}}{\|\mathbf{H}\|}$ is an isotropic unit vector along the direction of the channel $\mathbf{H}$. We mention here that $\|\mathbf{H}\|$ and $\phi$ are independent owing to the fact that the $H_{i}$ are i.i.d random variables. The mutual information between $Y^{\prime}$ and $X$ in this case is given by

$$
\begin{aligned}
\mathbb{I}\left(X ; Y^{\prime} \mid \mathbf{H}\right)= & \mathrm{E}_{\mathbf{H}} \mathrm{E}_{\boldsymbol{\Lambda}}\left[\mathbb{H}\left(Y^{\prime}\right)\right]-\mathrm{E}_{\mathbf{H}} \mathrm{E}_{\boldsymbol{\Lambda}}\left[\mathbb{H}\left(Y^{\prime} \mid X, \mathbf{H}\right)\right] \\
= & \mathrm{E}_{\mathbf{H}} \mathrm{E}_{\boldsymbol{\Lambda}}\left[\mathbb{H}\left(\|\mathbf{H}\| X+\boldsymbol{\phi}^{\dagger} \Lambda \mathbf{N}\right)\right]- \\
& \mathrm{E}_{\mathbf{H}} \mathrm{E}_{\boldsymbol{\Lambda}}\left[\mathbb{H}\left(\boldsymbol{\phi}^{\dagger} \Lambda \mathbf{N}\right)\right] .
\end{aligned}
$$

Since $\mathbf{N}$ is Gaussian, $\mathbb{I}\left(X ; Y^{\prime} \mid \mathbf{H}\right)$ will be maximized when $X$ is Gaussian. This directly yields (Equation 4)

$$
\begin{aligned}
C_{\text {adhoc }}(d) & =\mathrm{E}_{\Lambda} \mathrm{E}_{\mathbf{H}} \log _{2}\left[1+\frac{\frac{P}{d}\|\mathbf{H}\|^{2}}{\left\|\boldsymbol{\phi}^{\dagger} \Lambda\right\|^{2}}\right] \\
& =\mathrm{E}_{\boldsymbol{\Lambda}} \mathrm{E}_{\mathbf{H}} \log _{2}\left[1+\frac{\frac{P}{d} \sum_{n=1}^{d}\left|H_{n}\right|^{2}}{\sum_{m=1}^{d}\left|\phi_{m}\right|^{2}\left|\Lambda_{m}\right|^{2}}\right] .
\end{aligned}
$$

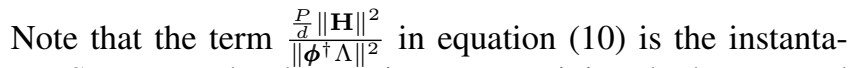
neous SINR seen by the receiver. Determining the best spread involves maximizing the capacity expression in equation (10) with respect to the user spread $d$. We now consider two extreme cases, low SINR $(S I N R<<1)$ and high SINR (SINR $>>1$ ) and analyze the optimal user spreads in these cases. We begin with the low SINR case in Theorem 1.

Theorem 1: At low SINR, the capacity of the link without interference knowledge at the receiver is maximized when the transmitter performs frequency hopping over single slots, i.e, $d^{*}=1$.

Proof: At low SINR, we have $\log (1+S I N R) \approx$ $S I N R$. From equation (10), the capacity of the user reduces to

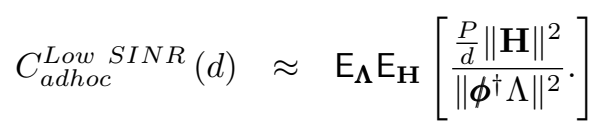

Since $\mathbf{H}=\|\mathbf{H}\| \boldsymbol{\phi}$ consists of i.i.d Rayleigh $H_{i},\|\mathbf{H}\|$ and $\phi$ are independent. The capacity can be expressed as

$$
\begin{aligned}
C_{\text {adhoc }}^{\text {Low } \operatorname{SINR}(d)} & =\mathrm{E}_{\|\mathbf{H}\|}\left[\frac{1}{d}\|\mathbf{H}\|^{2}\right] \mathrm{E}_{\Lambda} \mathrm{E}_{\boldsymbol{\phi}}\left[\frac{P}{\left\|\boldsymbol{\phi}^{\dagger} \Lambda\right\|^{2}}\right] \\
& =\mathrm{E}_{H_{1}}\left[\left|H_{1}\right|^{2}\right] \mathrm{E}_{\Lambda} \mathrm{E}_{\boldsymbol{\phi}}\left[\frac{P}{\sum_{m=1}^{d}\left|\phi_{m}\right|^{2}\left|\Lambda_{m}\right|^{2}}\right] .
\end{aligned}
$$

Note that $\phi$ is a unit vector, i.e., $\sum_{m=1}^{d}\left|\phi_{m}\right|^{2}=1$. Also, the $\Lambda_{i}$ are independent. Since $\frac{1}{x}$ is convex, we can write

$$
\begin{aligned}
C_{\text {adhoc }}^{\text {Low } \operatorname{SINR}(d)} & \leq \mathrm{E}_{\Lambda} \mathrm{E}_{\phi}\left[\sum_{m=1}^{d}\left|\phi_{m}\right|^{2} \frac{P \mathrm{E}_{H_{1}}\left[\left|H_{1}\right|^{2}\right]}{\left|\Lambda_{m}\right|^{2}}\right] \\
& =P \mathrm{E}_{H_{1}}\left[\left|H_{1}\right|^{2}\right] \mathrm{E}_{\Lambda_{1}}\left[\frac{1}{\left|\Lambda_{1}\right|^{2} \mid}\right] \\
& =C_{\text {adhoc }}^{\text {Low } \operatorname{SINR}(1) .}
\end{aligned}
$$

This directly yields the result of Theorem 1.

Theorem 1 therefore shows that hopping with $d=1$ outperforms spreading at low SINR. We now consider the high SINIR scenario. At high SINR, we have $\frac{\frac{P}{d}\|\mathbf{H}\|^{2}}{\left\|\boldsymbol{\phi}^{\dagger} \Lambda\right\|^{2}}>>1$ and the capacity reduces to

$$
\begin{aligned}
C_{\text {adhoc }}^{\text {High SINR }} & \approx \mathrm{E}_{\mathbf{H}} \mathrm{E}_{\boldsymbol{\Lambda}} \log \left[\frac{\frac{P}{d}\|\mathbf{H}\|^{2}}{\left\|\boldsymbol{\phi}^{\dagger} \Lambda\right\|^{2}}\right] \\
& =\mathrm{E}_{\mathbf{H}} \log \left[\frac{P}{d}\|\mathbf{H}\|^{2}\right]-\mathrm{E}_{\boldsymbol{\phi}} \mathrm{E}_{\boldsymbol{\Lambda}} \log \left[\left\|\boldsymbol{\phi}^{\dagger} \Lambda\right\|^{2}\right] .
\end{aligned}
$$

Note that $\mathrm{E}_{\mathbf{H}} \log \left[\frac{P}{d}\|\mathbf{H}\|^{2}\right] \leq \log \left[P \mathrm{E}_{\mathbf{H}}\left[\left|H_{1}\right|^{2}\right]\right]$. This might lead one to believe that spreading $(d \rightarrow \infty)$ is optimal. However note that the second term $T(d)=$ 
$\mathrm{E}_{\phi} \mathrm{E}_{\Lambda} \log \left[\left\|\phi^{\dagger} \Lambda\right\|^{2}\right]$ also depends on $d$. We now compare $T(d)$ and $T(d+1)$ and show that the second term is in fact an increasing function of $d$. In the following discussion, $\phi$ will be written as $\phi^{(d)}=\left[\begin{array}{llll}\phi_{1}^{(d)} & \phi_{2}^{(d)} & \cdots & \phi_{d}^{(d)}\end{array}\right]$ to explicitly show the dependence of $\phi$ on $d$. We have

$$
\begin{gathered}
T(d)=\mathrm{E}_{\boldsymbol{\phi}^{(d)}} \mathrm{E}_{\boldsymbol{\Lambda}} \log \left[\sum_{n=1}^{d}\left|\phi_{n}^{(d) \dagger} \Lambda_{n}\right|^{2}\right] \\
T(d+1)=\mathrm{E}_{\boldsymbol{\phi}^{(d+1)}} \mathrm{E}_{\boldsymbol{\Lambda}} \log \left[\sum_{n=1}^{d+1}\left|\phi_{n}^{(d+1) \dagger} \Lambda_{n}\right|^{2}\right]
\end{gathered}
$$

Note that both $\phi^{(d)}$ and $\phi^{(d+1)}$ are isotropic unit vectors. Consider all the realizations of $\phi^{(d+1)}$ where $\phi_{d+1}^{(d+1)}=0$. We have

$$
\mathrm{E}_{\boldsymbol{\phi}^{(d+1)}} \mathrm{E}_{\boldsymbol{\Lambda}}\left[\log \left[\sum_{n=1}^{d+1}\left|\phi_{n}^{(d+1) \dagger} \Lambda_{n}\right|^{2}\right] \mid \phi_{d+1}^{(d+1)}=0\right]=T(d) .
$$

Therefore $T(d)$ is an increasing function in $d$. The optimal spread for this case therefore depends on the relative increase of the two terms in $C_{\text {adhoc }}^{\text {High SINR }}$ with $d$. For Rayleigh $\mathbf{H}$ with $\mathrm{E}_{H_{i}}\left[\left|H_{i}\right|^{2}\right]=1$ and Poisson $\lambda_{i}$, contrary to the low SINR case, our numerical results suggest that at high SINR, spreading over all the available slots is optimal.

\section{NUMERICAL RESUltS}

We have already shown in Section IV that spreading the signal across the entire bandwidth of interest ensures that the user capacity is maximized in centralized scenarios. In

Capacity vs. $\alpha$ for $\mathrm{K}_{\mathrm{avg}}=32, \mathrm{~T}=128$ (Underloaded)

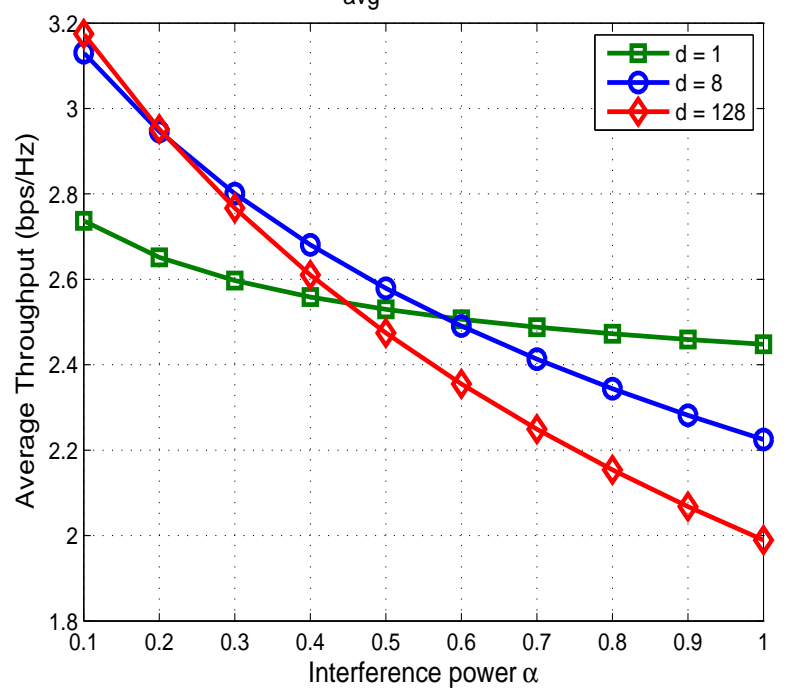

Fig. 2: Average user throughput (in bps/Hz) vs $\alpha$ for $d=1$, $d=1,8$ and 128 for an under-loaded system with $K_{a v g}=$ 32 and $T=128$. $d=1$ corresponds to pure hopping while $d=128$ corresponds to pure spreading. this section, we consider the ad-hoc case and provide numerical results based on Monte Carlo simulations for the best spreading strategies that maximize the user's throughput. We denote the average number of users in the system by $K_{\text {avg }}$ and consider a frequency selective channel with $T=128$ slots. We assume that the slots chosen by the user have independent and identically distributed Rayleigh fading channels with $\mathrm{E}\left[\left\|H_{i}\right\|^{2}\right]=1 . \lambda_{i}$ is assumed to be Poisson distributed with a mean of $\frac{K_{a v g}-1}{T}$. We look at the optimal spreads in underloaded $\left(K_{a v g}<<T\right)$, critically loaded $\left(K_{a v g}=T\right)$ and overloaded $\left(K_{\text {avg }}>>T\right)$ cases. The effect of the distance of the interferers from the user, reflected in the parameter $\alpha$ is also investigated. We will assume a power constraint $P=10$ for all our simulations.

Figure 2 plots the capacity of the system with increasing $\alpha$ for $d=1,8$ and 128 for an underloaded system $\left(K_{\text {avg }}=32\right.$ users, $T=128$ slots). As seen from the figure, as $\alpha$ increases from 0.1 to 1 , the optimal spread $d^{*}$ changes from $d^{*}=$ $T=128$ (complete spreading) to $d^{*}=1$ (complete hopping). This is consistent with Theorem 1 - for the underloaded case considered, $\alpha=1$ corresponds to a low SINR scenario. An interesting observation from Figure 2 is that there exist cases where the optimal strategy is neither complete spreading or complete hopping but some combination of the two. For example, for $0.25 \leq \alpha \leq 0.55$, spreading over $d=8$ slots performs better than complete spreading $(d=128)$ or pure hopping $(d=1)$.

Capacity vs. $\alpha$ for $\mathrm{K}_{\text {avg }}=128, T=128$ (Critically Loaded)

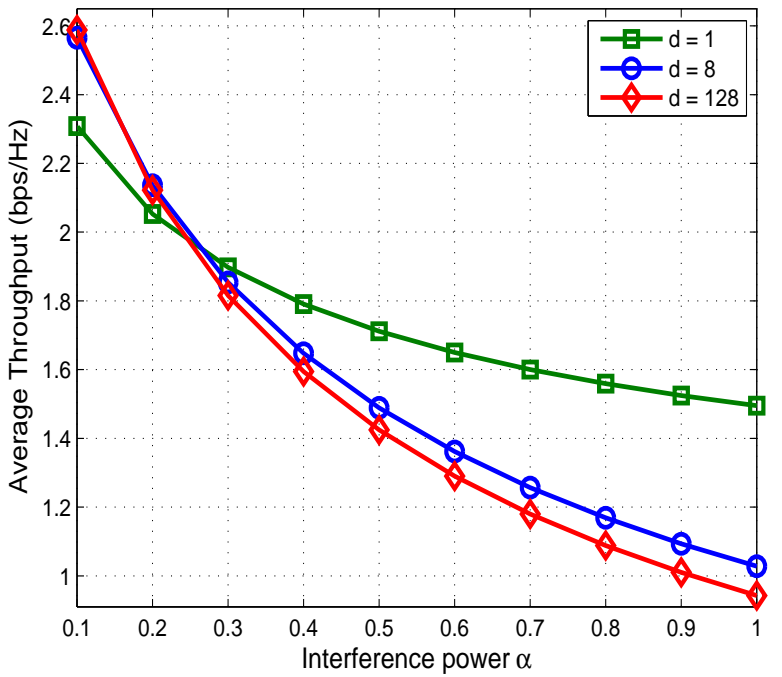

Fig. 3: Average user throughput (in bps/Hz) vs $\alpha$ for $d=1$, $d=1,8$ and 128 for a critically loaded system with $K_{a v g}=$ 128 and $T=128) . d=1$ corresponds to pure hopping while $d=128$ corresponds to pure spreading.

Figure 3 shows how capacity changes with $\alpha$ for a critically loaded system $\left(K_{a v g}=128\right.$ users, $T=128$ slots). Again as expected, the best spread goes from complete spreading to complete hopping as $\alpha$ increases. However the range where $d=8$ performs better than either pure spreading or hopping is smaller $(0.2 \leq \alpha \leq 0.25)$. Similar patterns are exhibited in 
the overloaded case.

Capacity vs. user spread for $\mathrm{K}_{\mathrm{avg}}=32, \mathrm{~T}=128$ (Underloaded)

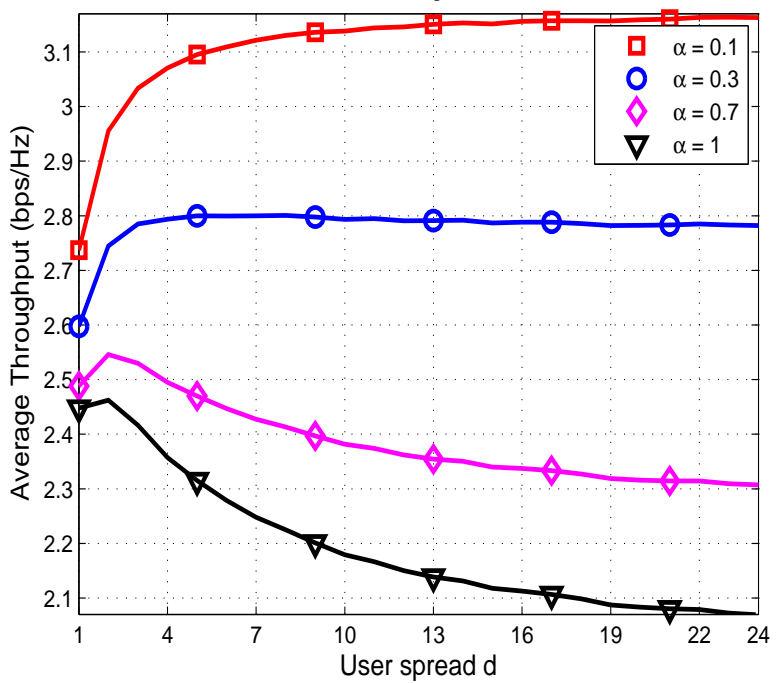

Fig. 4: Average user throughput (in $\mathrm{bps} / \mathrm{Hz}$ ) vs the user spread $d$ for different $\alpha$ (Underloaded system: $K_{a v g}=32, T=128$ ).

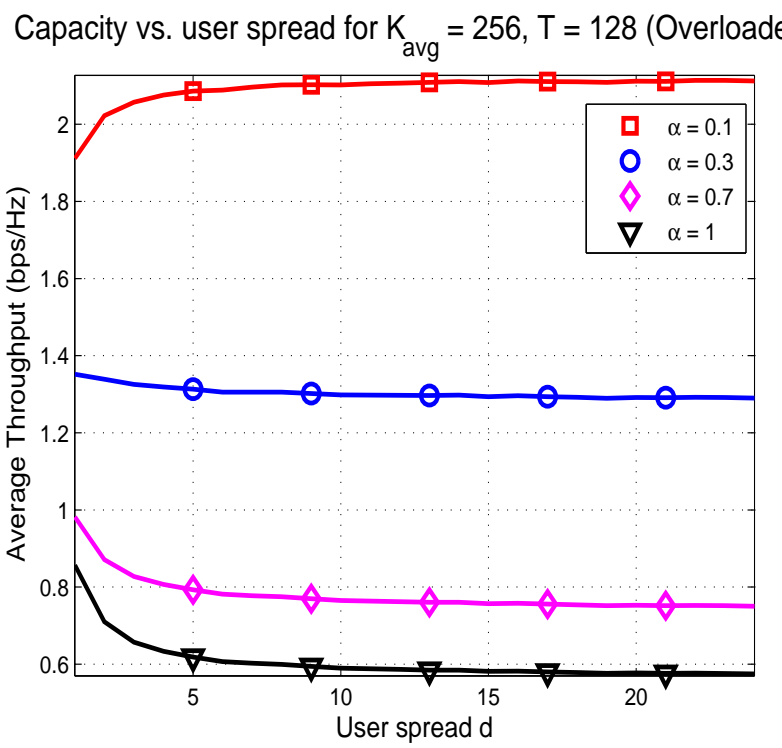

Fig. 5: Average user throughput (in bps/Hz) vs the user spread $d$ for different $\alpha$ (Critically loaded system: $K_{a v g}=128, T=$ 128).

We now plot the user capacity against $d$ to determine the optimal number of slots $d^{*}$ the user will have to spread over to maximize its throughput. Figure 4 compares the average throughput of an underloaded system with a total of $K_{\text {avg }}=$ 32 users in the $T=128$ slots against the user spread $d$. The throughput for up to $d=24$ slots are shown for the sake of clarity. As $\alpha$ (interference power) decreases, the capacity of the user for any spread $d$ decreases as expected. However, note that depending on the value of $\alpha$, the optimal spread may be neither $d=1$ or $d=T=128$. For example, when $\alpha=0.3$, the optimal spread $d^{*}=6$, i.e., capacity is maximized when the user spreads the signal over six independent slots and hops to a different set of six slots every transmission.

Figure 5 shows the optimal spreading strategies for the overloaded case ( $K_{\text {avg }}=128$ users and $T=128$ slots). The optimal spreads here are $d^{*}=1$ for low SINR and $d^{*}=T$ for high SINR.

\section{CONCLUSION}

We investigate the hopping-spreading tradeoff shown to arise from the logarithmic relation of the capacity on the SINR. While spreading is seen to average both the channel and the interference, hopping provides the receiver with varying channel and interference powers. Therefore neither spreading nor hopping satisfies both the capacity maximizing requirements of a constant channel and varying interference. Considering an ad-hoc link where the receiver has only channel and SINR information, we analyze this tradeoff and examine the optimal user spreads required to achieve the highest throughput in such scenarios. We prove that at low SINRs single slot frequency hopping is better than spreading. As a comparison we also demonstrate that spreading yields higher capacities than hopping in interference aware centralized situations. Monte Carlo simulations also show that there exist some cases where a hybrid spreading-hopping technique can provide higher data rates than either complete hopping or spreading. In ad-hoc systems spreading is also found to perform better than hopping at high SINRs.

\section{REFERENCES}

[1] Steven Weber, Xiangying Yang, Jeffrey G. Andrews and Gustavo De Veciana, "Transmission Capacity of Wireless Ad Hoc Networks with Outage Constraints," IEEE Transactions on Information Theory, December 2005.

[2] Steven Weber, Jeffrey G. Andrews, Xiangying Yang and Gustavo De Veciana, "Transmission Capacity of Wireless Ad Hoc Networks with Successive Interference Cancellation," Submitted to the IEEE Transactions on Information Theory, August 2005.

[3] Venugopal V. Veeravalli and Behnaam Aazhang, "On the CodingSpreading Tradeoff in CDMA Systems," Conference on Information Sciences and Systems, Princeton University, p. 11361141, March 1996.

4] Venugopal V. Veeravalli and Ashok Mantravadi, "The Coding-Spreading Tradeoff in CDMA Systems," IEEE Journal on Selected Areas in Communications, vol. 20, February 2002.

[5] C. Zheng and Muriel Médard, "How Far Should We Spread Using DSCDMA in Time and Frequency Selective Fading Channels?," Globecom Communication Theory Workshop, December 2003.

[6] Muriel Médard and David N.C. Tse, "Spreading in Block-Fading Channels," Asilomar Conference on Signals, Systems and Computers, 2000.

[7] John H. Gass Jr., Daniel L. Noneaker and Michael B. Pursley, "A Comparison of Slow-Frequency-Hop and Direct-Sequence Spread-Spectrum Packet Communications over Doubly Selective Fading Channels,' IEEE Transactions on Communications, vol. 50, pp. 1236 - 1239, August 2002

[8] John H. Gass Jr. and Michael B. Pursley, "Tradeoffs between FrequencyHop and Direct-Sequence Signaling for Frequency-Selective Fading Channels," Military Communications Conference, vol. 1, pp. 21-24, October 1996.

[9] Stavros Toumpis and Andrea J. Goldsmith, "Capacity Regions for Wireless Ad Hoc Networks," IEEE Transactions on Wireless Communications, vol. 2, July 2003.

[10] I. Emre Telatar, "Capacity of Multi-antenna Gaussian Channels," European Transactions on Telecom, vol. 10, pp. 585-596, November 1999.

[11] Andrea J. Goldsmith, Wireless Communications. Cambridge University Press, 2005.

[12] Thomas M. Cover and Joy A. Thomas, Elements of Information Theory. Wiley, 1991. 